\title{
Podocyte-specific Transcription Factors: Could MafB become a Therapeutic Target for Kidney Disease?
}

\author{
Naoki Morito, Toshiaki Usui, Shun Ishibashi and Kunihiro Yamagata
}

\begin{abstract}
:
The increasing number of patients with chronic kidney disease (CKD) is being recognized as an emerging global health problem. Recently, it has become clear that injury and loss of glomerular visceral epithelial cells, known as podocytes, is a common early event in many forms of CKD. Podocytes are highly specialized epithelial cells that cover the outer layer of the glomerular basement membrane. They serve as the final barrier to urinary protein loss through the formation and maintenance of specialized foot-processes and an interposed slit-diaphragm. We previously reported that the transcription factor MafB regulates the podocyte slit diaphragm protein production and transcription factor Tcf21. We showed that the forced expression of MafB was able to prevent CKD. In this review, we discuss recent advances and offer an updated overview of the functions of podocyte-specific transcription factors in kidney biology, aiming to present new perspectives on the progression of CKD and respective therapeutic strategies.
\end{abstract}

Key words: podocyte, transcription factor, chronic kidney disease, MafB, focal segmental glomerulosclerosis

(Intern Med Advance Publication)

(DOI: 10.2169/internalmedicine.9336-22)

\section{Introduction}

Chronic kidney disease (CKD) is highly prevalent in many countries, increasing the risk of cardiovascular disease as well as end-stage kidney disease (ESKD) (1). The number of ESKD patients requiring renal replacement therapy is increasing worldwide and becoming a major social burden. CKD has become a global public health problem, and effective therapeutic targets are urgently required to slow CKD progression and improve the prognosis (2).

Podocytes are highly specialized epithelial cells that cover the outer layer of the glomerular basement membrane (GBM). They serve as the final barrier to urinary protein loss through the formation and maintenance of specialized foot-processes and an interposed slit diaphragm (3). Because podocytes are highly differentiated cells, their injury and loss can only partially be compensated for by hypertrophy of the remaining podocytes (4).

Focal segmental glomerulosclerosis (FSGS) is a syndrome resulting from podocyte injury characterized by segmental sclerotic lesions in the glomeruli, cellular hypertrophy, loss of foot processes, pseudocyst formation, and changes of the microvilli. Podocyte loss or dysfunction is closely related to the development of FSGS lesions, proteinuria, and loss of nephrons (5,6) (Fig. 1). Many inheritable genetic forms of FSGS have been described, caused by mutations in genes whose products are mainly expressed in podocytes and which have important roles in the maintenance of the glomerular function. These proteins are mainly those regulating slit diaphragm structures, the actin cytoskeleton, foot process structures, and transcription factors in podocytes (7). Several transcription factors responsible for both podocyte differentiation and morphogenesis contribute to the development of proteinuria due to podocyte dysregulation.

We recently reported that transcription factor MafB is expressed in podocytes and that its mutation results in FSGS in humans and mice. Consistent with this, forced MafB expression was able to prevent CKD $(8,9)$. In the present report, we summarize relevant studies and highlight recent data on MafB and its role in renal biology, aiming to provide insights into potential areas for further investigation. We also review the presence and functions of other podocyte-specific transcription factors that might be viable 


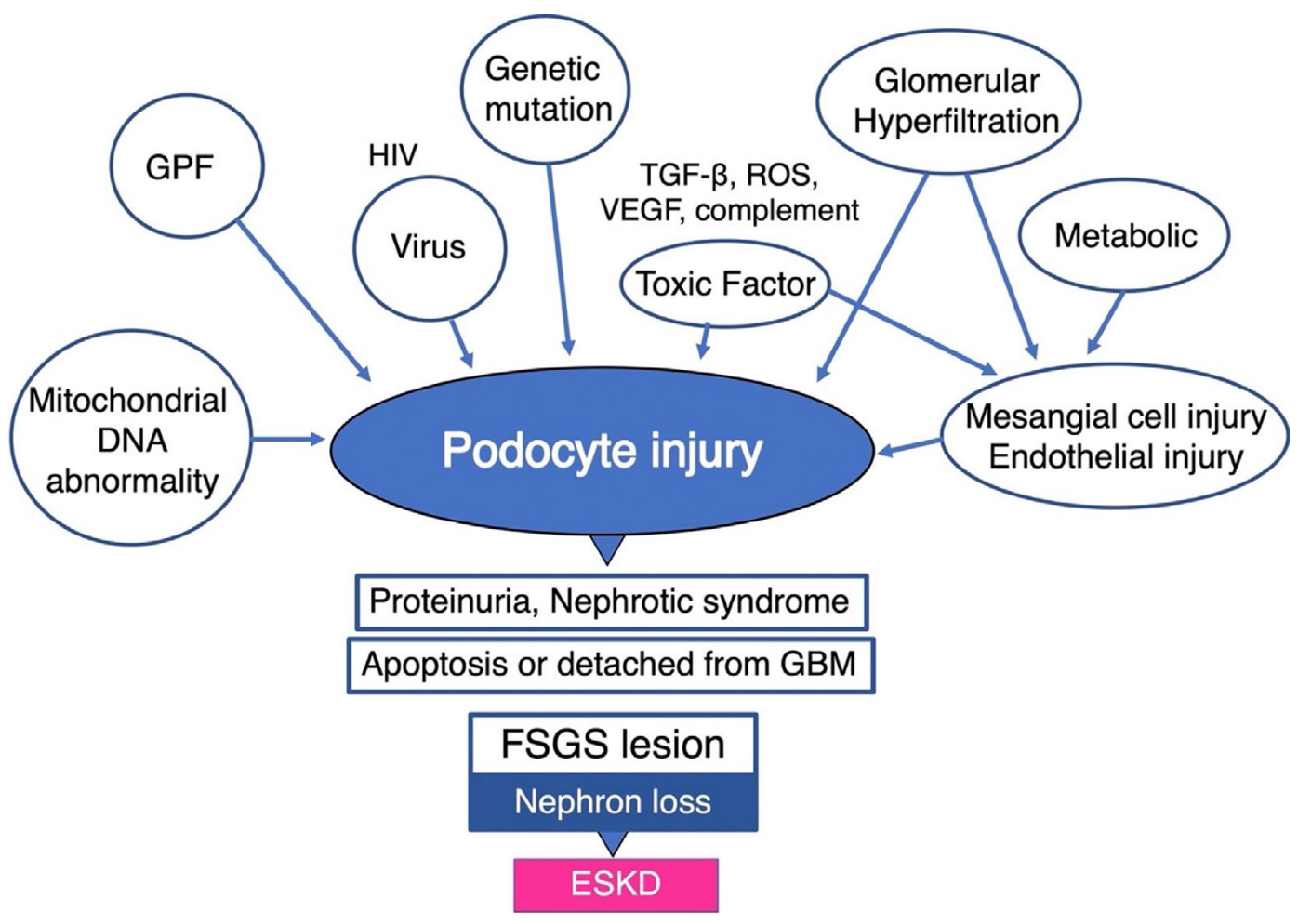

Figure 1. Schematic model of the podocyte injury pathogenesis. Podocyte injury leads to the development of FSGS lesion, proteinuria, and loss of nephron. GPF: Glomerular Permeability Factor, ESKD: End stage kidney disease, HIV: human immunodeficiency virus, ROS: Reactive oxygen species, FSGS: focal segmental glomerulosclerosis

as alternative therapeutic targets in CKD.

\section{Podocyte-specific transcription factor MafB}

\section{Large Maf proteins and MafB}

Members of the Maf transcription factor family are homologs of the v-maf oncogene, which was identified from the AS42 virus and causes Musculoaponeurotic fibrosarcoma in chicks (10). Maf family proteins share a conserved basic region and leucine zipper (bZip) motif that mediates dimer formation and DNA binding to the Maf recognition element (MARE) (11-13). The MAF family is divided into two subfamilies: large Maf transcription factors with a bZip domain and a transcriptional activation domain, and small Maf transcription factors with only a bZip domain $(11,13,14)$. In mammals, four large Maf proteins (MafA, MafB, c-Maf, and Nrl) have been identified (15) (Fig. 2). MafB is known to be essential for the development of pancreatic endocrine cells, formation of the inner ear, and functional differentiation of macrophages and osteoclasts (16). In addition, the $\mathrm{t}(14 ; 20)(\mathrm{q}$ $32 ; \mathrm{q} 11) / I G H-M A F B$ translocation is found in multiple myeloma, accounting for $1 \%-2 \%$ of cases (17). In kidneys, MafB is expressed in podocytes (18).

\section{Role of MafB in kidney development}

To elucidate the MafB function, we created MafBknockout (KO) mice. These animals died soon after birth due to hindbrain and renal abnormalities and respiratory failure (18). In newborn MafB-KO kidneys, the population of mature glomeruli was significantly reduced, and podocyte foot-process effacement was observed. A significant reduction in nephrin, podocin, and CD2AP expression, which is required for slit-diaphragm formation of glomeruli, was observed in MafB-KO mice. We thus concluded that MafB is essential for podocyte differentiation and foot process formation (18).

\section{The MafB function in podocytes}

Because mice deficient in conventional MafB die soon after birth (18), it is impossible to analyze the MafB function in adults. To overcome this hurdle, we generated conditional podocyte-specific $M a f B-K O$ (cKO) mice to analyze the MafB function in adults. MafB-cKO mice developed FSGS characterized by the depletion of slit diaphragm-related proteins (Nphs1 and Magi2) and deficiency in podocyte-specific transcription factor Tcf21(8). Nephrin, mutations of which are a major cause of congenital nephrotic syndrome, is specifically located at the slit-diaphragm of glomerular podocytes (19). Membrane-associated guanylate kinase, WW, and PDZ domain-containing 2 (Magi2) is also known to cause congenital nephrotic syndrome (20).

Although the relationships between TCF21 and human FSGS are not clear, $T c f 21$ podocyte-specific conditional knockout mice do develop FSGS (21). Liang et al. reported that spermidine is a key regulator in maintaining a high 




Transactivation domain

Extended Homology Region

Basic Region

Leucin Zipper

Figure 2. Structural features of Maf proteins. Schematic diagrams of four large Maf and three small Maf protein structures. Large Maf proteins contain a transactivation domain, but the small Maf proteins not. There are two forms of human c-Maf, as the result of differential splicing.

level of basal autophagy in podocytes (22). They examined the role of ornithine decarboxylase (ODC) and spermidine synthase (SRM) in autophagy. MafB is an upstream regulatory transcription factor of spermidine synthesis in podocytes, regulating the expression of ODC and SRM. Autophagy in turn regulates MafB, forming a positive feedback loop (22). In the diabetic state, MafB overexpression might ameliorate nephropathy through Nephrin, Gpx3, and Notch2 induction in podocytes (9). Gpx3, a member of the glutathione peroxidase family, can protect cells against oxidative stress (23). The Notch2 pathway plays a critical role in protecting damaged podocytes from apoptosis (24) (Fig. 3).

\section{MAFB mutations in human disease}

Because $M a f B$-deficient mice die during the perinatal period, it was suspected that $M A F B$ deficiency-related human diseases would not exist. However, in the past decade, human $M A F B$ mutations have been reported in patients with multicentric carpotarsal osteolysis (MCTO) and Duane retraction syndrome (DRS) (25-27).

MCTO is a rare skeletal dysplasia characterized by aggressive osteolysis, particularly affecting the carpal and tarsal bones. Because all carpal bones are present in MCTO patients during childhood, with osteolysis progressively occurring as patients age, it was hypothesized that this syndrome is caused by excessive bone resorption $(28,29)$. MCTO patients have mutations in the phosphorylation sites of the MAF transcriptional activation domain and exhibit abnormal phosphorylation (30) (Fig. 4A). The mechanisms responsible for osteoclast disease as a result of the $M A F B$ mutation are unknown. MCTO cases are reportedly complicated by hereditary FSGS, which is characterized by podocyte injury (31-33). To analyze the pathogenesis of this syndrome, we used CRISPR/Cas9 genome editing to create homozygous mice harboring the MCTO mutation. These animals manifested FSGS similar to that seen in MCTO patients (34).

Park et al. reported that $M A F B$ mutations cause DRS, a congenital eye movement disorder characterized by abnormal abduction (26). However, the authors did not report any renal involvement in their case. Recently, we and collaborators found that DRS patients carrying a $M A F B$ mutation in the DNA-binding domain p. Leu239Pro developed FSGS (27). To validate the identified mutation as the direct cause of disease, the same mutation was introduced into mice by genome editing. Mice homozygous for the DRS mutation exhibited impaired podocyte differentiation resembling that seen in MafB-deficient mice $(27,35)$.

Five DRS mutations in $M A F B$ have been reported $(26,27,36)$. Four are deletions, and the remaining one is a missense variant in the DNA-binding domain that renders binding to the MARE sequence impossible (Fig. 4B). Although MCTO and DRS with FSGS animals displayed the same renal phenotype, the extra-renal manifestations differed markedly, manifesting as osteoclast dysfunction or abducens nerve paralysis. Therefore, it is important 


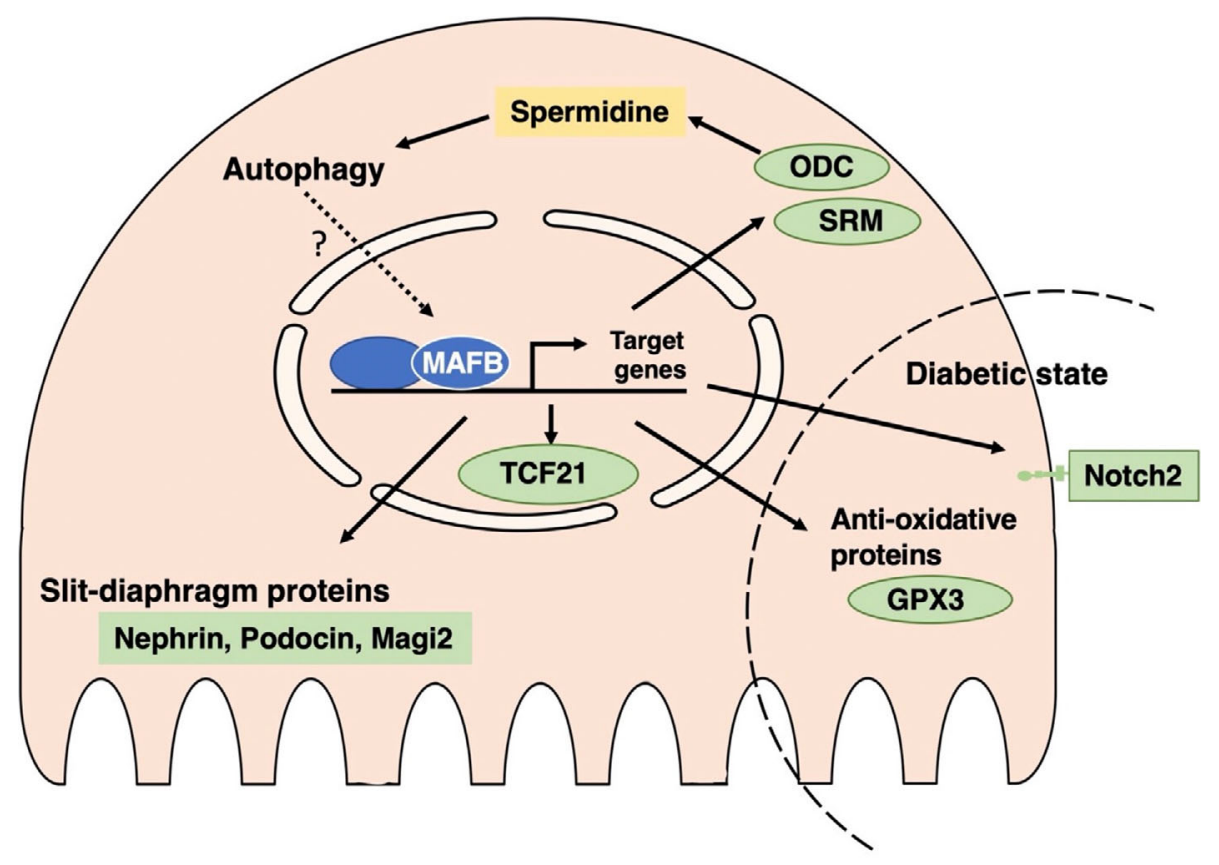

Figure 3. Schematic model of the MafB function in podocytes. MafB directly regulates slit-diaphragm proteins and transcription factor Tcf21. Schematic displaying the role of MafB in the interplay of autophagy and polyamine metabolism in podocytes. In diabetic states, Gpx3 and Notch2 are induced by MafB.

to analyze these mutant mice in greater detail in order to understand the pathological mechanisms involved.

\section{Role of MafB in CKD}

To analyze the relationship between MAFB and primary glomerular diseases, and diabetic kidney disease (DKD), we performed MAFB immunofluorescence studies in a variety of biopsy specimens (minimal change disease, IgA nephropathy, primary FSGS and DKD). We found a significantly lower MAFB expression in primary FSGS and DKD patients than minimal change disease and IgA nephropathy people. Primary FSGS is presumably caused by a circulating factor, possibly a cytokine produced from extrarenal sources, which causes generalized injury to podocytes (37). Similarly, in $\mathrm{DKD}$, podocytes seem to be highly susceptible to injury, which leads to their loss by apoptosis or detachment from the GBM (38). In experimental murine models of FSGS and $\mathrm{DKD}$, the MAFB expression in podocytes was decreased, as in human disease $(8,9)$. These results imply that MAFB plays a crucial role in FSGS and DKD pathogenesis. Consistent with this, streptozotocin-induced DKD was ameliorated by MafB overexpression in the podocytes of MafB podocyte-specific transgenic mice. Furthermore, MafBenforced overexpression, or treatment with a MafB inducer, protected against adriamycin-induced FSGS in mice (8). Thus, MafB may be a new therapeutic target for CKD.

\section{Transcription factors of podocytes related to FSGS}

The transcription factor mutations described below result in FSGS (Table) and may also be related to FSGS progression.

\section{Wilms tumor 1 (WT1)}

The WT1 gene encodes a zinc finger DNA-binding protein that acts as a transcriptional activator or repressor. It is vital for kidney development and is highly expressed in mature podocytes (39). WT1 regulates the expression of MafB, Lmxlb, Foxcl/2, and Tcf21, which are podocyte-specific transcription factors expressed by mature podocytes (40). Various different mutations of the WT1 gene have been identified as causes of syndromic hereditary FSGS (41). Mutations within the c-terminal zinc finger domains encoded by exons 8 and 9 have been shown to impair the transactivating functions of WT1. This results in significant alterations in the expression of genes, the products of which are essential slit diaphragm components, such as nephrin, podocin, and podocalyxin $(39,42-46)$. Renal phenotypes associated with WT1 mutations include Wilms' tumor as a component of WAGR syndrome (Wilms' tumor, aniridia, genitourinary anomalies and mental retardation), Denys-Drash syndrome (Wilms' tumor, male pseudohermaphroditism and early onset nephrotic syndrome with FSGS), and Frasier syndrome (male pseudohermaphroditism, nephrotic syndrome with FSGS histology and development of gonadoblastoma) $(42,47-51)$. WT1 mutations are found in approximately $8 \%-13 \%$ of patients with congenital nephrotic syndrome (52).

\section{Paired Box gene 2 (PAX2)}

PAX2 is a transcription factor playing a central role dur- 
A

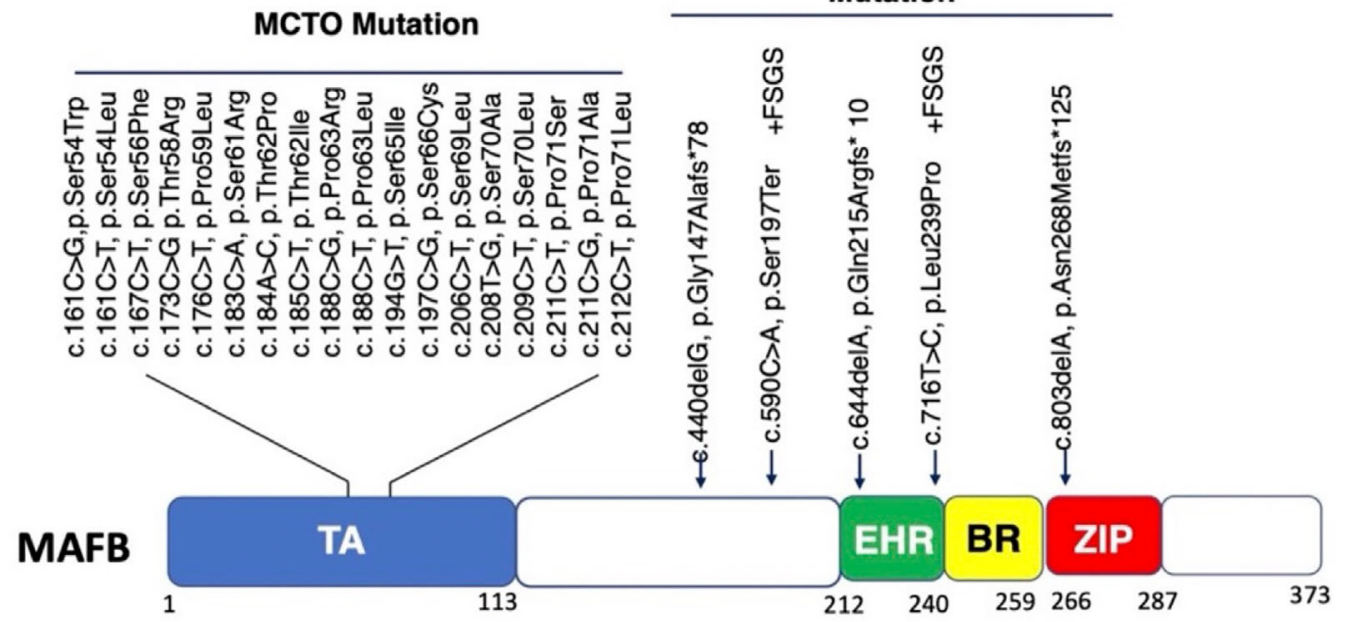

TA; Transactivation domain, EHR; Extended Homology Region, BR; Basic Region ZIP; Leucin Zipper

B

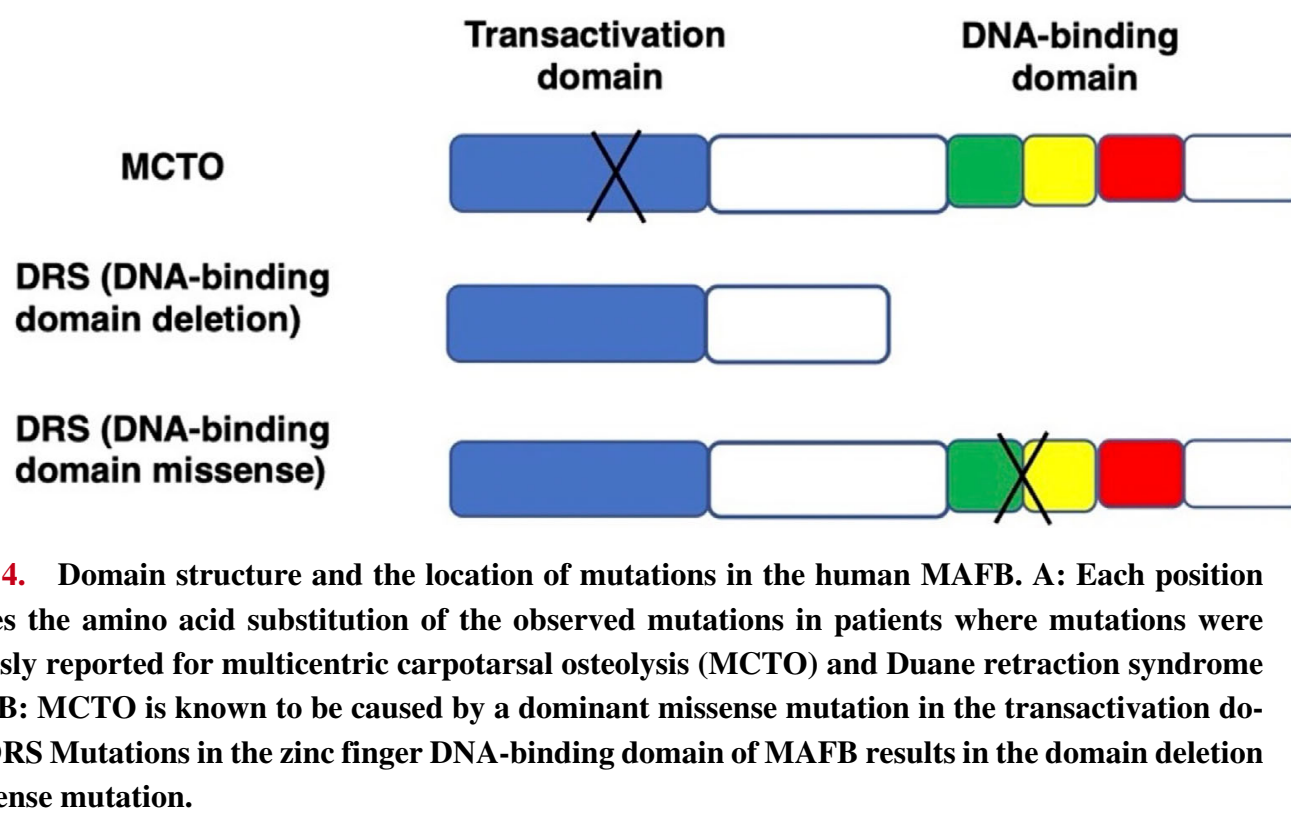

ing early embryonic kidney development. Heterozygous mutations in PAX2 result in papillorenal syndrome, also known as renal-coloboma syndrome. This is a rare autosomaldominant disease characterized by renal hypodysplasia, optic nerve abnormalities, and deafness (53). PAX2 mutations can also cause FSGS through haploinsufficiency and dominant negative effects (54). PAX2 mutations were found in $8 \%$ of congenital abnormalities of the kidney and urinary tract (CAKUT) cases and in $4 \%$ of adult-onset familial FSGS cases (54).

\section{LIM homeodomain transcription factor 1 (LMX1B)}

Mutations in the $L M X 1 B$ gene cause nail-patella syndrome (NPS). This is an autosomal-dominant disease characterized by dysplastic nails and elbows, hypoplastic or ab- sent patellae, iliac horns, and nephropathy (55). LMX1B mutants without extrarenal manifestations are recognized as LMX1B-associated nephropathy $(56,57)$. LMX1B contains two LIM-domains (LIM-A and LIM-B), which are involved in protein-protein interactions, and a homeodomain, which interacts with specific DNA elements in target genes (58). The genes encoding podocin, CD2AP and the $\alpha 3$ and $\alpha 4$ chains of collagen IV appear to be natural target gene candidates for LMX1B. Hence, $L M X 1 B$ mutation results in FSGS $(59,60)$. In patients with NPS, the prevalence of nephropathy is $25 \%(61)$. Electron microscopy typically demonstrates focal or diffuse irregular thickening of the GBM with electron-lucent areas. Some patients with NPS progress to ESKD (58). 
Table. Transcription Factors in Genetic FSGS.

\begin{tabular}{|c|c|c|c|c|}
\hline Gene & Protein & List of disease & $\begin{array}{c}\text { Renal } \\
\text { phenotype }\end{array}$ & $\begin{array}{l}\text { Mode of } \\
\text { inheritance }\end{array}$ \\
\hline$M A F B$ & $\begin{array}{l}\text { v-maf musculoaponeurotic } \\
\text { fibrosarcoma oncogene homolog B }\end{array}$ & MCTO Duane retraction syndrome & FSGS & $\mathrm{AD}$ \\
\hline WT1 & Wilms tumor 1 & $\begin{array}{c}\text { Denys-Drash syndrome Fraiser } \\
\text { syndrome }\end{array}$ & $\begin{array}{l}\text { FSGS } \\
\text { DMS }\end{array}$ & $\mathrm{AD}$ \\
\hline PAX2 & Paired box gene 2 & Renal-coloboma syndrome & $\begin{array}{l}\text { FSGS } \\
\text { CAKUT }\end{array}$ & $\mathrm{AD}$ \\
\hline$L M X 1 B$ & $\begin{array}{l}\text { LIM homeobox transcription factor } 1 \\
\text { beta }\end{array}$ & Nail-patella syndrome & FSGS & $\mathrm{AD}$ \\
\hline SMARCALI & $\begin{array}{l}\text { SWI/SNF-related matrix-associated } \\
\text { actin-dependent regulator of } \\
\text { chromatin subfamily A-like protein } 1\end{array}$ & $\begin{array}{l}\text { Schimke immuno-osseous } \\
\text { dysplasia }\end{array}$ & FSGS & AR \\
\hline NXF5 & Nuclear RNA export factor 5 & $\begin{array}{l}\text { X-linked familial FSGS with } \\
\text { co-segregating heart block disorder }\end{array}$ & FSGS & XR \\
\hline
\end{tabular}

MCTO: multicentric carpotarsal osteolysis, FSGS: focal segmental glomerulosclerosis, DMS: diffuse mesangial sclerosis, CAKUT: congenital anomalies of the kidney and urinary tract, AD: autosomal dominant, AR: autosomal recessive, and XR: X-linked recessive

4. SWI / SNF-related, matrix-associated, actindependent regulator of chromatin, subfamily a like 1 (SMARCAL1)

SMARCAL1, a member of the SWI2/SNF2 protein family, stabilizes replication forks during DNA damage. Schimke immuno-osseous dysplasia (SIOD) is a rare autosomal recessive disorder characterized by spondyloepiphyseal dysplasia with disproportionate growth failure, typical facial appearance, nephrotic syndrome with FSGS and progressive renal failure, recurrent lymphopenia, T-cell immunodeficiency, and pigment naevi $(62,63)$. Whole-exome sequencing of 300 families with steroid-resistant nephrotic syndrome revealed SMARCAL1 mutations in 8 families (64). Although SMARCAL1 is required for DNA double-strandbreak repair (65), the mechanisms linking SMARCAL1 mutation to podocytopathy are not clear at present.

\section{Nuclear RNA export factor 5 (NXF5)}

NXF5 is a member of the nuclear RNA export factor family. There is one report that an Australian pedigree carrying an NXF5 mutation manifested X-linked familial FSGS with co-segregating heart block disorder (66).

\section{Podocyte-specific transcription factors: Any association with FSGS?}

Although the transcription factors described below have not been reported as mutated in human FSGS, they play important roles in podocytes and may therefore have some relationship with FSGS.

\section{Kruppel-like factor (KLF) 4}

KLFs are zinc finger transcription factors involved in various cellular processes, such as cell differentiation, apoptosis, and proliferation (67). Hayashi et al. reported that Klf4 is highly expressed in glomerular podocytes, and its expression is decreased in individuals with proteinurea in both animal models and humans. Podocyte-specific Klf4-KO mice were shown to be particularly susceptible to adriamycin-induced FSGS (68). Klf4 overexpression in cultured podocytes increased the expression of nephrin and other epithelial markers and reduced mesenchymal gene expression. These investigators reported that Klf4 epigenetically modulates the podocyte phenotype and function and that the podocyte epigenome can be targeted for direct intervention and reduction of proteinuria (68). It was also reported that Klf4 expression in podocytes is required to maintain parietal epithelial cell (PEC) quiescence through podocyte-PEC crosstalk. Targeting podocyte-PEC crosstalk might be a useful therapeutic strategy in proliferative glomerulopathies (69).

\section{2, Transcription factor 21 (TCF21)}

TCF21 (POD1/capsulin/epicardin) is a basic helix-loophelix (bHLH) transcription factor whose expression is highest in podocyte precursors and is maintained in mature podocytes. Maezawa et al. reported that podocyte-specific $T c f$ 21-KO mice develop massive proteinuria and lesions similar to FSGS (70). In addition, Usui et al. reported that TCF21 is present in not only the nuclei but also the cytoplasm of injured podocytes. Therefore, TCF21 overexpression may have functional effects in injured podocytes (71).

\section{FOXC1 and FOXC2 (FOXC1/2)}

FOXC1 and FOXC2 (FOXC1/2) belong to subgroup C of the Forkhead-box (FOX) transcription factor superfamily involved in the development of many organs (72). In humans, mutations of FOXC1 are responsible for Axenfeld-Rieger syndrome, and mutations in FOXC2 underlie lymphedemadistichiasis syndrome $(73,74)$. Foxc1/2 regulate and stabilize podocyte-specific gene transcription, including the expression of Tcf7 12 and Klf6, which have been linked to kidney disease progression and podocyte damage (75). The kidneys of conditional-Foxc1/2-null mice showed proteinaceous casts, protein reabsorption droplets in tubules, and huge vacuoles in podocytes, indicating severe podocyte in- 
jury and massive proteinuria with FSGS lesions (76).

\section{Dachshund homolog 1 (DACH1)}

DACH1, a key cell-fate determinant, regulates transcription by DNA sequence-specific binding. DACH1 expression is reportedly reduced in human FSGS glomeruli (77). Global Dach1-KO mice manifest renal hypoplasia and die perinatally. Podocyte-specific Dachl-KO mice, however, maintain a normal glomerular architecture at baseline but rapidly develop podocyte injury after diabetes onset (78). In DKD, diminished DACH1 expression intensifies vulnerability to podocyte injury via epigenetic derepression of multiple DACH1 target genes. Furthermore, podocyte-specific augmentation of DACH1 expression in mice protects them from DKD. Podocyte-specific Dach1-KO mice are also extremely sensitive to ADR-induced FSGS (78).

\section{Summary}

In this review, we summarized the diverse roles of MafB and elaborated on its protective effects in kidney disease, providing new insights into progression and therapy for CKD. The identification of direct targets of MafB may result in the establishment of new therapeutic approaches for proteinuric renal diseases, including FSGS and DKD. In addition, we reviewed recent knowledge on other podocytespecific transcription factors, which can also represent therapeutic targets. Further clarification of the mechanisms underlying the development of CKD in relation to these transcription factors is warranted.

The authors state that they have no Conflict of Interest (COI).

\section{Acknowledgement}

The authors wish to thank Dr. Satoru Takahashi, Professor of anatomy and embryology, University of Tsukuba, for his help in interpreting the significance of the results of this study. This work was supported in part by the Ministry of Education, Culture, Sports, Science and Technology (MEXT) of Japan through Grants-in-Aid for Scientific Research (21K16157).

\section{References}

1. Levin A, Tonelli M, Bonventre J, et al. Global Kidney Health 2017 and beyond: a roadmap for closing gaps in care, research, and policy. Lancet 390: 1888-1917, 2017.

2. Hill NR, Fatoba ST, Oke JL, et al. Global prevalence of chronic kidney disease: a systematic review and meta-analysis. PLoS One 11: $\mathrm{e} 0158765,2016$.

3. Berger K, Moeller MJ. Podocytopenia, parietal epithelial cells and glomerulosclerosis. Nephrol Dial Transplant 29: 948-950, 2014.

4. Wiggins JE, Goyal M, Sanden SK, et al. Podocyte hypertrophy, "adaptation", and "decompensation" associated with glomerular enlargement and glomerulosclerosis in the aging rat: prevention by calorie restriction. J Am Soc Nephrol 16: 2953-2966, 2005.

5. Marshall CB, Shankland SJ. Cell cycle and glomerular disease: a minireview. Nephron Exp Nephrol 102: 39-48, 2006.

6. Wharram BL, Goyal M, Wiggins JE, et al. Podocyte depletion causes glomerulosclerosis: diphtheria toxin-induced podocyte de- pletion in rats expressing human diphtheria toxin receptor transgene. J Am Soc Nephrol 16: 2941-2952, 2005.

7. Fogo AB. Causes and pathogenesis of focal segmental glomerulosclerosis. Nat Rev Nephrol 11: 76-87, 2015.

8. Usui T, Morito N, Shawki HH, et al. Transcription factor MafB in podocytes protects against the development of focal segmental glomerulosclerosis. Kidney Int 98: 391-403, 2020.

9. Morito N, Yoh K, Ojima M, et al. Overexpression of Mafb in podocytes protects against diabetic nephropathy. J Am Soc Nephrol 25: 2546-2557, 2014.

10. Nishizawa M, Kataoka K, Goto N, Fujiwara KT, Kawai S. v-maf, a viral oncogene that encodes a "leucine zipper" motif. Proc Natl Acad Sci USA 86: 7711-7715, 1989.

11. Kataoka K, Noda M, Nishizawa M. Maf nuclear oncoprotein recognizes sequences related to an AP-1 site and forms heterodimers with both Fos and Jun. Mol Cell Bio 14: 700-712, 1994.

12. Yoshida T, Ohkumo T, Ishibashi $S$, Yasuda K. The 5'-AT-rich halfsite of Maf recognition element: a functional target for bZIP transcription factor Maf. Nucleic Acids Res 33: 3465-3478, 2005.

13. Motohashi H, Katsuoka F, Shavit JA, Engel JD, Yamamoto M. Positive or negative MARE-dependent transcriptional regulation is determined by the abundance of small Maf proteins. Cell 103: 865-875, 2000.

14. Igarashi K, Kataoka K, Itoh K, Hayashi N, Nishizawa M, Yamamoto M. Regulation of transcription by dimerization of erythroid factor NF-E2 p45 with small Maf proteins. Nature 367: 568-572, 1994.

15. Takahashi S. Functional analysis of large MAF transcription factors and elucidation of their relationships with human diseases. Exp Anim 70: 264-271, 2021.

16. Hamada M, Tsunakawa Y, Jeon H, Yadav MK, Takahashi S. Role of MafB in macrophages. Exp Anim 69: 1-10, 2020.

17. Stewart AK, Bergsagel PL, Greipp PR, et al. A practical guide to defining high-risk myeloma for clinical trials, patient counseling and choice of therapy. Leukemia 21: 529-534, 2007.

18. Moriguchi T, Hamada M, Morito N, et al. MafB is essential for renal development and F4/80 expression in macrophages. Mol Cell Biol 26: 5715-5727, 2006.

19. Kestilä M, Lenkkeri U, Männikkö M, et al. Positionally cloned gene for a novel glomerular protein--nephrin--is mutated in congenital nephrotic syndrome. Mol Cell 1: 575-582, 1998.

20. Ruotsalainen V, Ljungberg P, Wartiovaara $J$, et al. Nephrin is specifically located at the slit diaphragm of glomerular podocytes. Proc Natl Acad Sci USA 96: 7962-7967, 1999.

21. Bierzynska A, Soderquest K, Dean P, et al. UK study of Nephrotic Syndrome. MAGI2 Mutations Cause Congenital Nephrotic Syndrome. J Am Soc Nephrol 28: 1614-1621, 2017.

22. Liang W, Yamahara K, Hernando-Erhard C, et al. A reciprocal regulation of spermidine and autophagy in podocytes maintains the filtration barrier. Kidney Int 98: 1434-1448, 2020.

23. Reddy AT, Lakshmi SP, Banno A, Reddy RC. Role of GPx3 in PPARgamma-induced protection against COPD-associated oxidative stress. Free Radic Biol Med 126: 350-357, 2018.

24. Tanaka E, Asanuma K, Kim E, et al. Notch2 activation ameliorates nephrosis. Nat Commun 5: 3296, 2014.

25. Zankl A, Duncan EL, Leo PJ, et al. Multicentric carpotarsal osteolysis is caused by mutations clustering in the amino-terminal transcriptional activation domain of MAFB. Am J Hum Genet 90: 494-501, 2012.

26. Park JG, Tischfield MA, Nugent AA, et al. Loss of The MafB function in humans and mice causes Duane Syndrome, aberrant extraocular muscle innervation, and inner-ear defects. Am J Hum Genet 98: 1220-1227, 2016.

27. Sato $\mathrm{Y}$, Tsukaguchi $\mathrm{H}$, Morita $\mathrm{H}$, et al. A mutation in transcription factor MAFB causes Focal Segmental Glomerulosclerosis with Duane Retraction Syndrome. Kidney Int 94: 396-407, 2018. 
28. Klein C, Bellity J, Finidori G, Glorion C, Pannier S. Multicentric carpotarsal osteolysis syndrome: long-term follow-up of three patients. Skeletal Radiol 47: 1015-1019, 2018.

29. Zhuang L, Adler S, Aeberli D, Villiger PM, Trueb B. 2017. Identification of a MAFB mutation in a patient with multicentric carpotarsal osteolysis. Swiss Med Wkly 147: w14529, 2017.

30. Herath NI, Rocques N, Garancher A, Eychène A, Pouponnot C. 2014. GSK3-mediated MAF phosphorylation in multiple myeloma as a potential therapeutic target. Blood Cancer J 4: e175, 2014.

31. Connor A, Highton J, Hung NA, Dunbar J, MacGinley R, Walker R. Multicentric carpal-tarsal osteolysis with nephropathy treated successfully with cyclosporine A: a case report and literature review. Am J Kidney Dis 50: 649-54, 2007.

32. Park PG, Kim KH, Hyun HS, et al. Three cases of multicentric carpotarsal osteolysis syndrome: a case series. BMC Med Genet 19: 164, 2018.

33. Närhi A, Fernandes A, Toiviainen-Salo S, et al. A family with partially penetrant multicentric carpotarsal osteolysis due to gonadal mosaicism: First reported case. Am J Med Genet A 185: $2477-$ 2481, 2021.

34. Tsunakawa Y, Hamada M, Matsunaga Y, et al. Mice harboring an MCTO muta- tion exhibit renal failure resembling nephropathy in human patients. Exp Anim 68: 103-111, 2019.

35. Kanai M, Jeon H, Ojima M, et al. Phenotypic analysis of mice carrying human-type MAFB p.Leu239Pro mutation. Biochem Biophys Res Commun 523: 452-457, 2020.

36. Kaimori JY, Mori T, Namba-Hamano T, et al. Cyclosporine A Treatment of Proteinuria in a New Case of MAFB-Associated Glomerulopathy without Extrarenal Involvement: A Case Report. Nephron 145: 445-450, 2021.

37. De Vriese AS, Sethi S, Nath KA, Glassock RJ, Fervenza FC. Differentiating Primary, Genetic, and Secondary FSGS in Adults: A Clinicopathologic Approach. J Am Soc Nephrol 29: 759-774, 2018.

38. Pagtalunan ME, Miller PL, Jumping-Eagle S, et al. Podocyte loss and progressive glomerular injury in type II diabetes. J Clin Invest 99: 342-348, 1997.

39. Ratelade J, Arrondel C, Hamard G, et al. A murine model of Denys-Drash syndrome reveals novel transcriptional targets of WT 1 in podocytes. Hum Mol Genet 19: 1-15, 2010.

40. White JT, Zhang B, Cerqueira DM, Tran U, Wessely O. Notch signaling, wt1 and foxc2 are key regulators of the podocyte gene regulatory network in Xenopus. Development 137: 1863-1873, 2010.

41. Kaltenis $P$, Schumacher V, Jankauskiene A, Laurinavicius A, Royer-Pokora B. Slow progressive FSGS associated with an F392 L WT1 mutation. Pediatr Nephrol 19: 353-356, 2004.

42. Mucha B, Ozaltin F, Hinkes BG, et al. Mutations in the Wilms' tumor 1 gene cause isolated steroid resistant nephrotic syndrome and occur in exons 8 and 9. Pediatr Res 59: 325-331, 2006.

43. Palmer RE, Kotsianti A, Cadman B, Boyd T, Gerald W, Haber DA. WT1 regulates the expression of the major glomerular podocyte membrane protein Podocalyxin. Curr Biol 11: 1805-1809, 2001.

44. Guo G, Morrison DJ, Licht JD, Quaggin SE. WT1 activates a glomerular-specific enhancer identified from the human nephrin gene. J Am Soc Nephrol 15: 2851-2856, 2004.

45. Gao F, Maiti S, Sun G, et al. The Wt1+/R394W mouse displays glomerulosclerosis and early-onset renal failure characteristic of human Denys-Drash syndrome. Mol Cell Biol 24: 9899-9910, 2004.

46. Morrison AA, Viney RL, Saleem MA, Ladomery MR. New insights into the function of the Wilms tumor suppressor gene WT1 in podocytes. Am J Physiol Renal Physiol 295: F12-F17, 2008.

47. Niaudet P, Gubler MC. WT1 and glomerular diseases. Pediatr Nephrol 21: 1653-1660, 2006.
48. Gbadegesin R, Hinkes BG, Hoskins BE, et al. Mutations in PLCE 1 are a major cause of isolated diffuse mesangial sclerosis (IDMS). Nephrol Dial Transplant 23: 1291-1297, 2008.

49. Chernin G, Vega-Warner V, Schoeb DS, et al. Genotype/phenotype correlation in nephrotic syndrome caused by WT1 mutations. Clin J Am Soc Nephrol 5: 1655-1662, 2010.

50. Royer-Pokora B, Beier M, Henzler M, et al. Twenty-four new cases of WT1 germline mutations and review of the literature: Genotype/phenotype correlations for Wilms tumor development. Am J Med Genet A 127A: 249-257, 2004.

51. Denamur E, Bocquet N, Mougenot B, et al. Mother-to-child transmitted WT1 splice-site mutation is responsible for distinct glomerular diseases. J Am Soc Nephrol 10: 2219-2223, 1999.

52. Sadowski CE, Lovric $\mathrm{S}$, Ashraf $\mathrm{S}$, et al. A single-gene cause in $29.5 \%$ of cases of steroid-resistant nephrotic syndrome. J Am Soc Nephrol 26: 1279-1289, 2015.

53. Sanyanusin P, Schimmenti LA, McNoe LA, et al. Mutation of the PAX2 gene in a family with optic nerve colobomas, renal anomalies and vesicoureteral reflux. Nature genetics 9: 358-364, 1995.

54. Barua M, Stellacci E, Stella L, et al. Mutations in PAX2 Associate with Adult-Onset FSGS. J Am Soc Nephrol 25: 1942-1953, 2014.

55. McIntosh I, Dreyer SD, Clough MV, et al. Mutation analysis of LMX1B gene in nail-patella syndrome patients. Am J Hum Genet 63: 1651-1658, 1998.

56. Boyer O, Woerner S, Yang F, et al. LMX1B mutations cause hereditary FSGS without extrarenal involvement. J Am Soc Nephrol 24: 1216-1222, 2013.

57. Shimohata H, Miyake Y, Yoshida Y, et al. LMX1B-associated nephropathy that showed myelin figures on electron microscopy. CEN Case Rep 10: 588-591, 2021.

58. Witzgall R. Nail-patella syndrome. Pflugers Arch 469: 927-936, 2017.

59. Morello R, Zhou G, Dreyer SD, et al. Regulation of glomerular basement membrane collagen expression by LMX1B contributes to renal disease in nail patella syndrome. Nat Genet 27: 205-208, 2001.

60. Heidet L, Bongers EM, Sich M, et al. In vivo expression of putative LMX1B targets in nail-patella syndrome kidneys. Am J Pathol 163: 145-155, 2003.

61. Ghoumid J, Petit F, Holder-Espinasse M, et al. Nail-Patella Syndrome: clinical and molecular data in 55 families raising the hypothesis of a genetic heterogeneity. Eur J Hum Genet 24: 44-50, 2016.

62. Boerkoel CF, Takashima H, John J, et al. Mutant chromatin remodeling protein SMARCAL1 causes Schimke immuno-osseous dysplasia. Nat Genet 30: 215-220, 2002.

63. Lücke T, Franke D, Clewing JM, et al. Schimke versus nonSchimke chronic kidney disease: an anthropometric approach. Pediatrics 118: e400-e407, 2006.

64. Warejko JK, Tan W, Daga A, et al. Whole Exome Sequencing of Patients with Steroid-Resistant Nephrotic Syndrome. Clin J Am Soc Nephrol 13: 53-62, 2018.

65. Keka IS, Mohiuddin, Maede Y, Rahman MM, et al. Smarcal1 promotes double-strand-break repair by nonhomologous end-joining. Nucleic Acids Res 43: 6359-6372, 2015.

66. Esposito T, Lea RA, Maher BH, et al. Unique X-linked familial FSGS with co-segregating heart block disorder is associated with a mutation in the NXF5 gene. Hum Mol Genet 22: 3654-3666, 2013.

67. Mallipattu SK, Estrada CC, He JC. The critical role of Kruppellike factors in kidney disease. Am J Physiol Ren Physiol 312: F 259-F265, 2017.

68. Hayashi K, Sasamura H, Nakamura M, et al. KLF4-dependent epigenetic remodeling modulates podocyte phenotypes and attenuates proteinuria. J Clin Investig 124: 2523-2537, 2014.

69. Pace JA, Bronstein R, Guo Y, et al. Podocyte-specific KLF4 is re- 
quired to maintain parietal epithelial cell quiescence in the kidney. Sci Adv 7: eabg6600, 2021.

70. Maezawa Y, Onay T, Scott RP, et al. Loss of the podocyteexpressed transcription factor Tcf21/Pod1 results in podocyte differentiation defects and FSGS. J Am Soc Nephrol 25: 2459-2470, 2014.

71. Usui J, Yaguchi M, Yamazaki S, et al. Transcription factor 21 expression in injured podocytes of glomerular diseases. Sci Rep 10: 11516,2020

72. Kaestner KH, Knochel W, Martinez DE. Unified nomenclature for the winged helix/forkhead transcription factors. Genes Dev 14: 142-146, 2000

73. Rice R, Rice DP, Olsen BR, Thesleff I. Progression of calvarial bone development requires Foxc1 regulation of Msx2 and Alx4. Dev Biol 262: 75-87, 2003.

74. Kume T, Deng KY, Winfrey V, Gould DB, Walter MA, Hogan BLM. The forkhead/winged helix gene Mf1 is disrupted in the pleiotropic mouse mutation congenital hydrocephalus. Cell 93:
985-996, 1998

75. Yang J, Zhang D, Motojima M, et al. Super-Enhancer-Associated Transcription Factors Maintain Transcriptional Regulation in Mature Podocytes. J Am Soc Nephrol 32: 1323-1337, 2021.

76. Motojima M, Kume T, Matsusaka T. Foxc1 and Foxc2 are necessary to maintain glomerular podocytes. Exp Cell Res 352: 265272, 2017.

77. Hodgin JB, Borczuk AC, Nasr SH, et al. A molecular profile of focal segmental glomerulosclerosis from formalin-fixed, paraffinembedded tissue. Am J Pathol 177: 1674-11686, 2010.

78. Cao A, Li J, Asadi M, Basgen JM, et al. DACH1 protects podocytes from experimental diabetic injury and modulates PTIP-H3K4 Me3 activity. J Clin Invest 131: e141279, 2021.

The Internal Medicine is an Open Access journal distributed under the Creative Commons Attribution-NonCommercial-NoDerivatives 4.0 International License. To view the details of this license, please visit (https://creativecommons.org/licenses/ by-nc-nd/4.0/).

\section{(C) The Japanese Society of Internal Medicine} Intern Med Advance Publication 